\title{
Periodic formation and propagation of double layers in the expanding chamber of an inductive discharge operating in $\mathrm{Ar} \mathrm{SF}_{6}$ mixtures
}

\author{
N. Plihon, ${ }^{\text {a) }}$ C. S. Corr, P. Chabert, and J.-L. Raimbault \\ Laboratoire de Physique et Technologie des Plasmas, Ecole Polytechnique, \\ 91128 Palaiseau Cedex, France
}

(Received 3 February 2005; accepted 16 May 2005; published online 25 July 2005)

\begin{abstract}
It has previously been shown [Tuszewski et al., Plasma Sources Sci. Technol. 12, 396 (2003)] that inductive discharges in electronegative gases are subject to two types of instability: the source instability related to the $E$ to $H$ transition and a transport instability, occurring downstream when an expanding chamber is present. These two types of instability are observed in our "helicon" reactor operated without a static magnetic field in low-pressure $\mathrm{Ar} / \mathrm{SF}_{6}$ mixtures. Temporally and spatially resolved measurements show that, in our experiment, the downstream instability is a periodic formation and propagation of a double layer. The double layer is born at the end of the source tube and propagates slowly to the end of the expansion region with a velocity of $150 \mathrm{~m} \mathrm{~s}^{-1}$. (C) 2005 American Institute of Physics. [DOI: 10.1063/1.1947387]
\end{abstract}

\section{INTRODUCTION}

Inductive reactors are routinely used for etching of silicon and various metals in the microelectronic industry. The plasma is excited by flowing a rf current in a coil which launches a decaying wave into the plasma through a dielectric window. When operating in the high plasma density regime (the inductive $H$ mode), they provide high ion fluxes with adjustable ion energies by means of an additional $\mathrm{rf}$ biasing of the wafer holder. However, when the power applied to the coil is low, the $H$ mode cannot be sustained and the discharge operates in a capacitive $(E)$ mode due to the high voltage across the coil with respect to the grounded walls. The transition between the $E$ mode and the $H$ mode (by increasing the input power) is the first source of instability of inductive reactors when electronegative gases are used. This phenomenon, referred as the source instability, has been described and modeled in a series of recent papers. ${ }^{1-8}$

When the inductive source sits on top of an expanding chamber, another type of instability has been experimentally identified by Tuszewski and co-workers. ${ }^{7,9}$ The instability is spatially localized in the diffusion chamber and has been termed the downstream instability. The linear stability analysis of a set of fluid equations shows that the transport becomes unstable if the difference between positive-ion and negative-ion fluid velocities exceeds a threshold. ${ }^{10}$ This threshold was also obtained using a kinetic theory. The nonlinear development of this instability was not theoretically studied but some features were experimentally emphasized. In this paper, we have experimentally investigated instabilities in a "helicon" reactor operated without a static magnetic field, i.e., in the inductive mode. The reactor has a cylindrical geometry with a source and a diffusion chamber of different radii. The source instability and a downstream instability, which will be compared to the one described by Tuszewski et $a l^{9}$ and Tuszewski and Gary, ${ }^{10}$ were identified. We will show that the downstream instability observed in our system

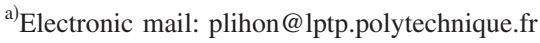

is due to the formation of a double layer, which propagates (slowly) into the diffusion chamber. The formation and propagation are periodic with a frequency in the kilohertz range.

The paper is organized as follows: Sec. II describes the experimental setup and diagnostics, Sec. III summarizes the characteristics of the two types of instability, while the downstream instability is fully investigated in Sec. IV. Concluding remarks and discussion are offered in Sec. V.

\section{EXPERIMENTAL ARRANGEMENT AND DIAGNOSTICS}

The reactor is shown schematically in Fig. 1. It consists of a source chamber sitting on top of a 32-cm-diameter diffusion chamber. This chamber is terminated by a movable plate, introduced through the bottom of the diffusion chamber, such that the diffusion chamber length can be varied between 0 and $26 \mathrm{~cm}$. We could also insert a $13-\mathrm{cm}$-innerdiameter aluminum cylinder in the diffusion chamber, so that the discharge geometry did not present any geometrical discontinuity and was a $13-\mathrm{cm}$-diameter, 56-cm-long cylinder.

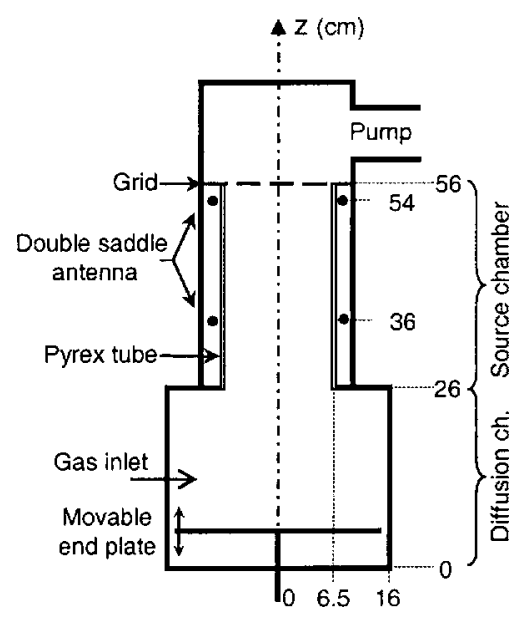

FIG. 1. Schematic of the experimental setup. 
The source is a $15-\mathrm{cm}$-diameter, 30-cm-long, and $0.9-\mathrm{cm}$ thick Pyrex cylinder surrounded by a double saddle-fieldtype helicon antenna. ${ }^{11}$ The fan-cooled antenna is powered through a close-coupled $L$-type matching network by a rf power supply operating at $13.56 \mathrm{MHz}$ and capable of delivering up to $2-\mathrm{kW}$ forward power. The time-averaged input power was recorded as the difference between the timeaveraged forward and reflected powers. The Pyrex cylinder is housed in an aluminum cylinder of 20-cm diameter and 30 $\mathrm{cm}$ long. A metal grid attached to the other end of the source tube confines the plasma and isolates it from a turbomolecular pump that routinely maintains base pressures of $10^{-6}$ mbar. The discharge was run in $\mathrm{Ar}$ and $\mathrm{Ar}: \mathrm{SF}_{6}$ mixtures. The partial gas pressures of $\mathrm{Ar}$ and $\mathrm{SF}_{6}$ were determined by controlling the flows.

The measurements reported here were made along the revolution axis ( $z$ axis) of the discharge. The plasma parameters are determined using two types of electrostatic probes. The first is a nickel planar probe having a guard ring biased at the same (negative) potential as the probe, to measure the real positive-ion saturated current. The diameter of the collecting area is $4 \mathrm{~mm}$ and the diameter of the outer ring is 8 $\mathrm{mm}$. The second is a passively compensated Langmuir probe, ${ }^{12}$ of 0.25 -mm-diameter and 6 -mm-long platinum wire tip. For stationary plasmas and time-averaged measurements during the instability, the plasma potential, the electron density, and the electron temperature were deduced from the $I(V)$ characteristics of the cylindrical probe using a SmartSoft data acquisition system. ${ }^{13}$ The electronegativity $\alpha=n_{-} / n_{e}$ and consequently the ion densities (electroneutrality $n_{+}=n_{-}+n_{e}$ was assumed) were measured according to the double-probe technique described in Ref. 14. This technique, which relies on the theory developed in Ref. 15, allows to deduce $\alpha$ from the ratio of the cylindrical probe current at the plasma potential to the positive-ion saturation current measured by the planar probe, $R=I\left(V_{p}\right) / I_{\text {sat+ }}$. It requires an estimation of the ratio of the electron temperature to the negative-ion temperature $\gamma=T_{e} / T_{-}$and the positive-ion mass $m_{+}$, both difficult to measure in the gas mixture studied here. We chose $\gamma=15$, as is commonly thought to be a reasonable value in low-pressure electronegative discharges, $m_{+}=40$ since (i) $\mathrm{Ar}^{+}$may be dominant since we used small percentages of $\mathrm{SF}_{6}$ in argon and (ii) we expect a fairly high dissociation degree of $\mathrm{SF}_{6}$ and therefore $\mathrm{SF}_{x}^{+}$ions with $x \ll 6$ (low mass ions). As a consequence of these estimations, the absolute values of $\alpha$ should be regarded as indicative. However, we believe that spatial gradients of $\alpha$ or relative variations with operating conditions (pressure, power, and mixture) are correctly captured by the technique.

To probe the charged-particle and potential dynamics during the instability cycles, we have developed a timeresolved technique using both the cylindrical Langmuir probe and a fast broadband spectrum photodiode. The photodiode collects a chamber-integrated emission signal used as a phase reference. A computer-controlled dc voltage supply is used to bias the probe at a given potential for a time span $T_{N}$, corresponding to $N$ instability periods ( $N$ being typically 10-50). During $T_{N}$, the photodiode current and the probe current (voltage across a resistor $R$ ) are simultaneously recorded on a large memory oscilloscope. The probe voltage is then increased by a fraction of volts and the acquisition starts again. A typical voltage scan lasts $30 \mathrm{~s}$ for an acquisition of 20 instability periods (a period being roughly $1 \mathrm{~ms}$ ) for 120 voltage steps to record $I(V)$ characteristics with a good resolution. At the end of the voltage scan, data is downloaded from the oscilloscope's memory to the computer via a general purpose interface bus (GPIB). A period renormalization routine is used to correct the natural frequency modulation of the instability (which could be up to 10\%). The technique allows to reconstruct the $I(V)$ probe characteristics for each time step during the instability period, which are then analyzed to obtain the plasma parameters. According to Ref. 16 we computed the second derivative of the $I(V)$ curves using a Savitzky-Golay (SG) filter ${ }^{17}$ of order 3, over 7-11 points depending on the operating conditions. Since the filter works only for equally spaced voltage points, we compute the derivatives with respect to the supply voltage and use $d^{2} I / d V_{\text {probe }}^{2}=d^{2} I / d V_{\text {supply }}^{2}\left(1-\left[R\left(d I / d V_{\text {supply }}\right)\right]\right)^{-3}$ (Ref. 18) to calculate the derivatives with respect to the probe voltage. The zero of the second derivative is used to determine the plasma potential, the electron density is then deduced from the probe current at that potential (i.e., the electron thermal current). The electronic temperature is computed by fitting $\log \left(I_{e}\right)=V / T_{e}+$ const by least-square means between the floating potential and the plasma potential (where $I_{e}$ is the probe current minus the ion current, which was considered to be $\propto \sqrt{V-V_{p}}$ as proposed by Ref. 19). The time-resolved system has been successfully tested against the SmartSoft system for a stable Ar plasma under a large set of operating conditions. The averaging procedure indeed filters highfrequency features, but correctly measures periodic phenomena occurring up to tens of kilohertz.

\section{INSTABILITY WINDOW AND FEATURE}

The discharge becomes unstable when operating above a few percent of $\mathrm{SF}_{6}$ added to argon. Fluctuations are observed in the electron density, the positive- and negative-ion densities, the plasma potential, and the electron temperature. Two types of instability were identified depending on the gas pressure and input power used: (i) source oscillations occurring in the neighborhood of the $E$ to $H$ transition and (ii) a downstream instability, which will be discussed in detail in Sec. IV. The global instability windows in the pressurepower plane (gas pressure when the plasma is off, input power), for a $50 \% \mathrm{SF}_{6}$ concentration plasma, are shown in Fig. 2 for the maximum diffusion chamber length (i.e., the terminating plate is at $z=0 \mathrm{~cm}$ ). Figure 2(a) was obtained for fixed matching conditions (such that the reflected power was minimal at $5 \mathrm{mTorr} / 500 \mathrm{~W}$ ), whereas Fig. 2(b) was obtained by minimizing the reflected power at each pressure/power condition. The discharge was found to be unstable over all of the operating conditions. The source instability window depends drastically upon the matching conditions as has been previously observed and modeled, ${ }^{2,5,20}$ whereas the downstream instability does not. The changes made to the source relaxation oscillations when matching the discharge are the following; (i) the window of existence is reduced and (ii) the 

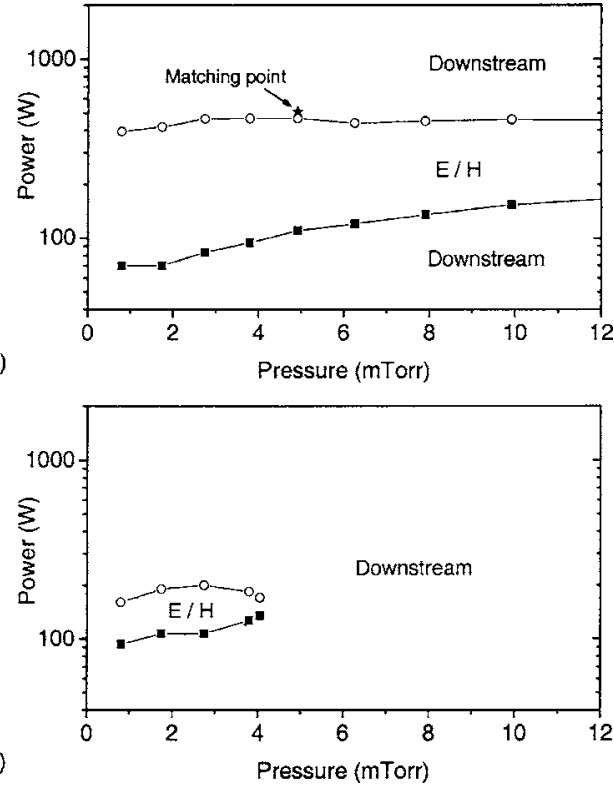

FIG. 2. The power and pressure windows of the instability for a $50 \% \mathrm{SF}_{6}$ concentration discharge when the end plate is at $z=0 \mathrm{~cm}$ (a) for one matching condition $(500 \mathrm{~W}, 5 \mathrm{mT})$ and (b) for best matching condition for each pressure/power.

amplitude of plasma parameter modulations is reduced by a factor of 4 . The characteristics of the downstream instability (amplitude and frequency) are not changed when changing the matching conditions.

Tuszewski et al. experimentally determined ${ }^{9}$ that their downstream instability could be suppressed when the length of the discharge was decreased. Hence, we measured instability windows when the terminating plate was at $z=26 \mathrm{~cm}$, i.e., with no diffusion chamber. These are shown in Fig. 3. The source instability remains almost similar to the case presented in Fig. 2: the area covered by the source instability is similar and is reduced when operating at best matching conditions. The downstream instability has been suppressed for all the operating conditions studied (1-12 mTorr and 0-2000 $\mathrm{W})$. When searching for the critical length $l_{c}$ of the diffusion chamber above which the downstream instability can develop, we found that $l_{c}$ is an increasing function of the pressure for $50 \% \mathrm{SF}_{6}$ concentration discharges $\left(l_{c}\right.$ increasing from $1.7 \mathrm{~cm}$ at $1 \mathrm{mTorr}$ to $3 \mathrm{~cm}$ at $3.5 \mathrm{mTorr}$ ).

To summarize, the source instability can (almost) be suppressed by carefully designing the rf circuit, including the matching unit, whereas the downstream instability is efficiently suppressed by removing the expanding region. These observations were already suggested by previous works. ${ }^{5,9}$ The results presented next have been obtained with the terminating plate at the bottom of the diffusion chamber at a pressure of 1 mTorr.

The spatiotemporal dynamics of the two instabilities are different. Figures 4 and 5 show the temporal variations of the positive-ion and the electron currents for the source (when the discharge is operated unmatched) and the downstream instabilities, respectively. The electron current was acquired by biasing the Langmuir probe (LP) at $+35 \mathrm{~V} ; 10 \mathrm{~mA}$ corresponds to an electron density of about $2 \times 10^{10} \mathrm{~cm}^{-3}$ for an electron temperature of $3 \mathrm{eV}$ (assuming that the plasma po-
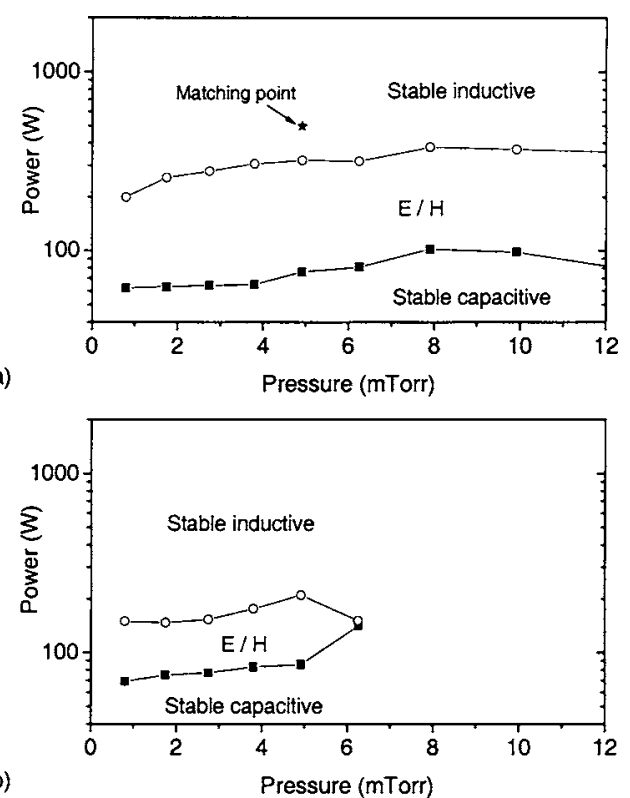

FIG. 3. The power and pressure windows of the instability for a $50 \% \mathrm{SF}_{6}$ concentration discharge when the end plate is at $z=26 \mathrm{~cm}$ i.e., without the diffusion chamber (a) for one matching condition (500 W, $5 \mathrm{mT})$ and (b) for best matching condition for each pressure/power.

tential is indeed $+35 \mathrm{~V}$, see Sec. IV for a discussion on plasma potential dynamics). The ion current is the current on the planar probe biased at $-50 \mathrm{~V} ; 1 \mathrm{~mA}$ corresponds to an ion density of $2.5 \times 10^{11} \mathrm{~cm}^{-3}$ when assuming that the Bohm velocity is $\left(k T_{e} / \gamma m_{+}\right)^{1 / 2}$, as for a highly electronegative plasma. ${ }^{26}$

Let us first consider the source instability presented in Fig. 4. The relaxation oscillations at around $650 \mathrm{~Hz}$ are strongest (largest amplitude) in the source chamber and decay on moving downstream into the diffusion chamber while remaining in phase. The rf current and the rf voltage, measured at the output of the rf generator, are also modulated at the instability frequency. The relaxation oscillations show an increasing frequency dependence with increasing pressure (from $700 \mathrm{~Hz}-1 \mathrm{kHz}$ at $1 \mathrm{mTorr}$ to $4-5 \mathrm{kHz}$ at 8 mTorr), as was already observed and modeled. ${ }^{2}$

The downstream instability oscillations (shown in Fig. 5) have lower amplitude than the source instability, with a maximum amplitude in the diffusion chamber, at a position which depends on power and pressure. In the source, the particle fluxes are only slightly modulated (the modulation being 5\%-20\% depending on the operating conditions), and appear to be in phase for all $z$ above $26 \mathrm{~cm}$. However, there may be a fast ion acoustic wave propagating upstream (at a speed of about $7000 \mathrm{~m} \mathrm{~s}^{-1}$ ), as described in Ref. 10. On the other hand, in the diffusion chamber, the oscillations do not remain in phase when moving downstream which clearly indicates a low-speed traveling phenomenon. The dynamics are the following: (i) at $t=0$ the densities decrease by $50 \%-$ $75 \%$ over a few microseconds, (ii) the densities are subject to large modulation in the $10-\mathrm{kHz}$ range over a few tens to a few hundreds of microseconds depending on the axial position, and (iii) an abrupt refilling front appears first at the top of the diffusion chamber and propagates slowly to the bottom plate. The frequency of occurrence of the downstream 

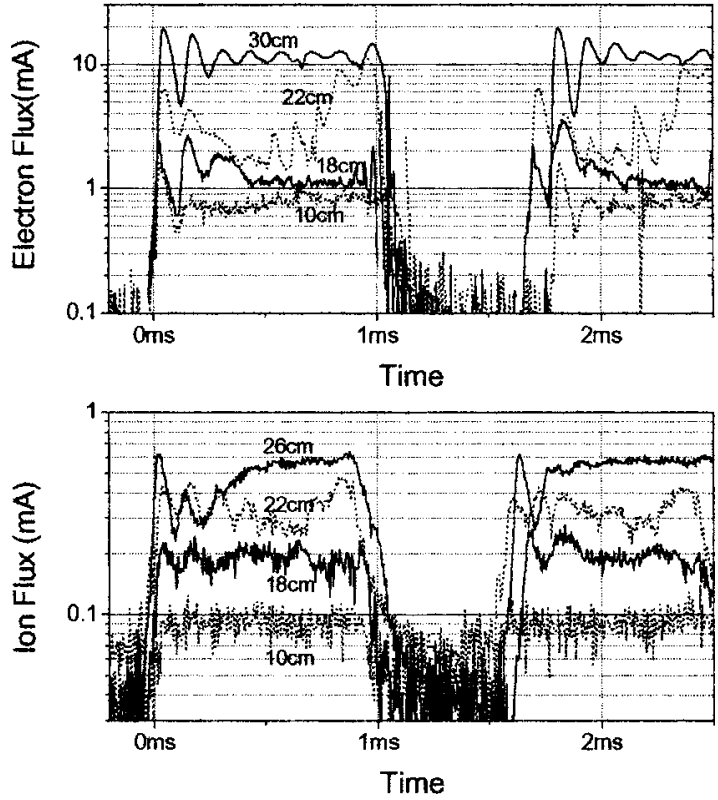

FIG. 4. Source instability: time dependence of the positive-ion saturation current (planar probe at $-50 \mathrm{~V}$ ) and electron flux (current collected by a LP biased at $+35 \mathrm{~V}$ ) for various axial positions for a $50 \% \mathrm{SF}_{6}$ concentration plasma, $1 \mathrm{~m}$ Torr, and input power of $250 \mathrm{~W}$ ( $100 \mathrm{~W}$ reflected). Signals are low-pass $(160 \mathrm{kHz})$ filtered.

instability is in the kilohertz range, with slight changes occurring when changing the pressure, power, or $\mathrm{SF}_{6}$ concentration. The spatio temporal evolution of the plasma parameters is presented in Sec. IV.

\section{DOWNSTREAM INSTABILITY WITH DOUBLE-LAYER FORMATION}

To further investigate the downstream instability we first measured the time-averaged plasma parameters along the $z$ axis in the diffusion chamber. The time-averaged plasma potential and negative-ion fraction profiles are shown in Fig. 6 for various $\mathrm{SF}_{6}$ percentages, with the input power being 600 $\mathrm{W}$ and the pressure 1 mTorr. For small $\mathrm{SF}_{6}$ concentrations, namely, below $8 \%$, the plasma is stationary and the plasma potential smoothly decays from the source to the diffusion chamber, as expected for an expanding plasma, while the negative-ion fraction is fairly constant $\left(\alpha \approx 3\right.$ for $6 \%$ of $\left.\mathrm{SF}_{6}\right)$. Between $8 \%$ and $13 \%$ of $\mathrm{SF}_{6}$, the plasma remains stationary but the plasma potential exhibits an abrupt drop of about $5 \mathrm{~V}$ at around $z=23 \mathrm{~cm}$, i.e., a double layer is formed. This double-layer formation is described in detail in Ref. 21, but the main features are the following. The double layer separates a high-density low-electronegativity plasma upstream from a low-density high-electronegativity plasma downstream. From visual observations, the double layer (DL) appears to be attached to the interface between the two chambers and expands into the diffusion chamber with a spherical shape (hence the DL crosses the axis at $z \approx 23 \mathrm{~cm}$ ). As the $\mathrm{SF}_{6}$ concentration is further increased, the discharge becomes unstable, entering the downstream instability regime, and one needs to use time-resolved measurements.

Using the time-resolved LP system described in Sec. II, we were able to measure the spatiotemporal evolution of the
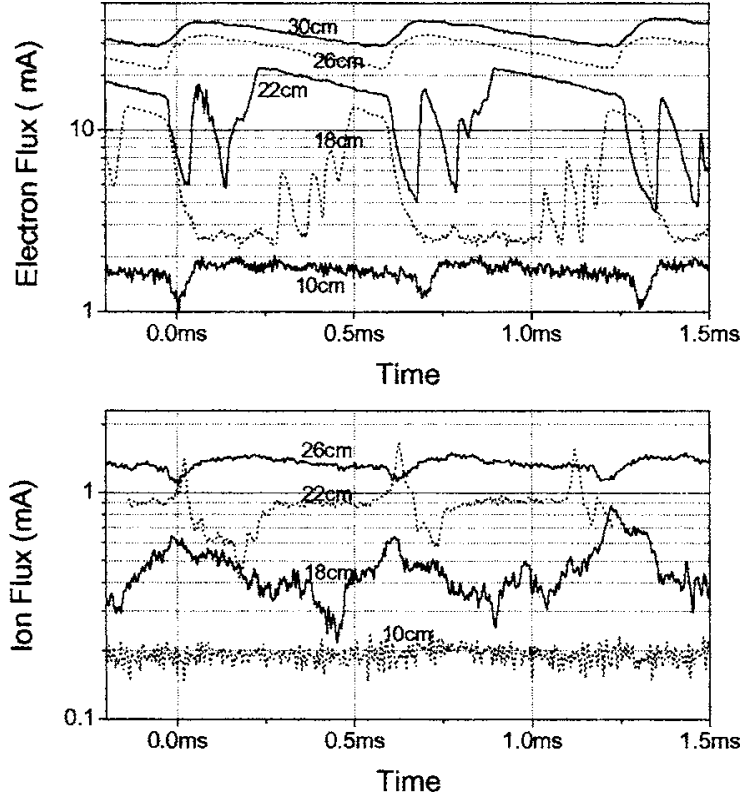

FIG. 5. Downstream instability: time dependence of the positive-ion saturation current (planar probe at $-50 \mathrm{~V}$ ) and electron flux (current collected by a LP biased at $+35 \mathrm{~V}$ ) for various axial positions for a $50 \% \mathrm{SF}_{6}$ concentration plasma, 1 mTorr, and input power of $600 \mathrm{~W}$ (30 W reflected). Signals are low-pass $(160 \mathrm{kHz})$ filtered.

plasma parameters. When operating with $50 \% \mathrm{SF}_{6}$ concentration, we observed (see Fig. 5) some density fluctuations in the $10-\mathrm{kHz}$ range just before the occurrence of the refilling front, which are not periodic and make difficult the processing of time-resolved probe signals. However, these $10-\mathrm{kHz}$ fluctuations were not observed for moderate $\mathrm{SF}_{6}$ concentrations (below 30\%).

Figure 7 shows the plasma potential measured on the reactor axis as a function of $z$, and as a function of time for a $25 \% \mathrm{SF}_{6}$ concentration plasma at $600 \mathrm{~W}$ and $1 \mathrm{mTorr}$. The $x$ axis is the time, normalized to the instability period, and the $y$ axis is the axial position (the diffusion chamber bottom

a)

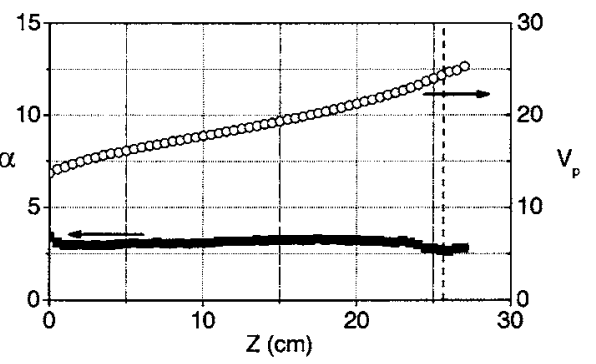

b)

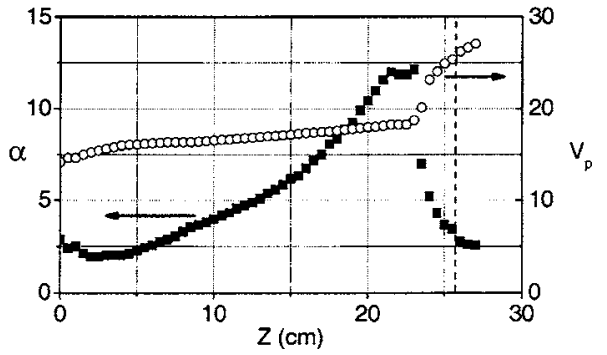

FIG. 6. Spatial evolution of the plasma potential and electronegativity in the diffusion chamber at $600 \mathrm{~W}$ and $1 \mathrm{~m}$ Torr, for different $\mathrm{SF}_{6}$ concentrations: (a) $6 \% \mathrm{SF}_{6}$ concentration, no DL case and (b) $9 \% \mathrm{SF}_{6}$ concentration, stable DL case. 



FIG. 7. Spatiotemporal evolution of the plasma potential, two instability periods for a $25 \% \mathrm{SF}_{6}$ concentration plasma at $600 \mathrm{~W}$ and 1 mTorr. A 3D representation and a gray level amplitude image are shown.

is at $z=0 \mathrm{~cm}$ ). For the given conditions the frequency of the oscillations is $770 \mathrm{~Hz}$, with frequency modulation being less than $2 \%$ (which allows the time-resolved proccessing described before to accurately measure the low-frequency phenomenon). Figure 7 shows two graphs: a 3D plot which is appropriate to clearly visualize the potential drop evolution, and a gray level image, useful to measure the propagation speed. The interface between the source and diffusion chambers is at $z=26 \mathrm{~cm}$ and is shown by a black dashed line in both graphs. It seems that the double layer is born at the interface between the two chambers and moves downward as time evolves. The potential drop is about $7 \mathrm{~V}$ at the beginning of the instability period and is decreasing as the DL propagates downward. The frequency of the double-layer formation and the propagation speed are such that the first double layer has not reached the bottom of the diffusion chamber when a new double layer forms upstream. Consequently, at a given time during the instability cycle, there are two potential drops in the diffusion chamber. The propagation speed is small, about $150 \mathrm{~m} / \mathrm{s}$, and mostly constant although the double layer appears to speed up slightly at the end of its travel when the new double layer is formed. This propagation speed is approximately the positive-ion thermal velocity. The plasma parameters are only slightly modulated in the source region, thus, it does not seem that the instability is driven by the source. Indeed, unlike for the source instability case, the rf current is not modulated. Electron densities can also be determined using the time-resolved measuring system. The electrons appear to be in Boltzmann equilibrium. At the beginning of the instability period, the electron density $n_{e}$ is about $1.5 \times 10^{11} \mathrm{~cm}^{-3}$ in the source, slowly decreases down to $6 \times 10^{10} \mathrm{~cm}^{-3}$ at the end of the source tube $(z=26 \mathrm{~cm})$, and experiences an abrupt drop at the doublelayer position, to reach $1 \times 10^{10} \mathrm{~cm}^{-3}$ for $z$ between 25 and $20 \mathrm{~cm}$. A second drop in the electron density occurs at the position of the second small double layer. Electron temperatures are higher on the high-potential side, with timeaveraged data giving $3 \mathrm{eV}$ at $z=10 \mathrm{~cm}$ and $4.5 \mathrm{eV}$ at $z$ $=26 \mathrm{~cm}$ and above.

Experimental evidence of a similar behavior for pressures between 1 and 10 mTorr has been drawn. Few changes were observed when increasing the pressure: (i) the plasma potential in the source is lowered when increasing the pressure (from $35 \mathrm{~V}$ at $1 \mathrm{mTorr}$ to $25 \mathrm{~V}$ at $8 \mathrm{mTorr}$ ) while the downstream time-averaged plasma potential remains roughly constant, hence the amplitude of the propagating double layer decreases with increasing pressure and (ii) the frequency increases with increasing pressure. However, when plotting the spatiotemporal evolution of the plasma potential as in Fig. 7 for various pressures, the slope of the white dash-dotted line remains constant, i.e., the propagation speed of the double layer normalized to the instability period remains constant.

Figure 7 shows that the double layer is born at the interface between the two chambers, as it was proposed for electropositive gases ${ }^{22}$ and was visually observed for the steady double-layer case in our system. ${ }^{21}$ We investigated the importance of the discontinuity in radius on the birth of the propagating double layer by inserting an inner tube in the diffusion chamber (hence resulting in a cylindrical discharge without geometrical discontinuity). New instability windows are plotted in Fig. 8; Fig. 8(a) was obtained for fixed matching conditions (such that the reflected power was minimal at $5 \mathrm{mTorr} / 500 \mathrm{~W}$ ), whereas Fig. 8(b) was obtained by minimizing the reflected power at each pressure/power condition. The stability regions are somewhat changed from the previous case: (i) the downstream instability window is drastically reduced, especially at pressure above 5 mTorr and (ii) two types of source oscillation are observed when operating with best matching conditions. The "strong $E / H$ " corresponds to the case previously described, where the modulation rate of the plasma parameters can reach nearly $100 \%$, and the "weak $E / H$ " corresponds to small ripples (5\%-10\% modulation) in the plasma parameters at a lower frequency than the strong fluctuations occuring at the same pressure when operating unmatched. Even though the stability of the discharge can be improved by the presence of the inner tube, there is still a window of operation where a double layer periodically forms and propagates. The temporal traces indicate that the formation of the DL occurs in the source at $z \approx 30 \mathrm{~cm}$.

\section{DISCUSSION AND CONCLUSION}

Our inductively coupled plasma discharge shows two kinds of instability when operating in $\mathrm{Ar} / \mathrm{SF}_{6}$ mixtures. The first appears at the transition between the low-density capacitive mode and the high-density inductive mode, and is 


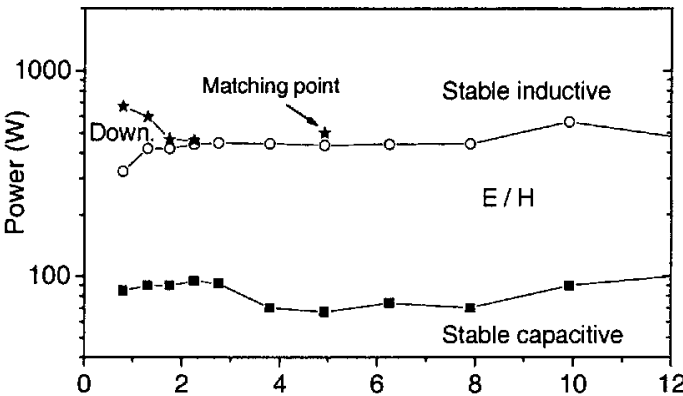

a)

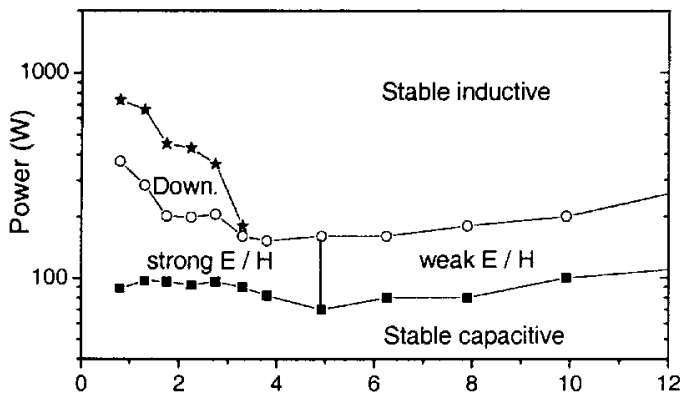

b)

Pressure (mTorr)

FIG. 8. The power and pressure windows of the instability for a $50 \% \mathrm{SF}_{6}$ concentration discharge when the inner tube has been placed in the diffusion chamber (a) for one matching condition $(500 \mathrm{~W}, 5 \mathrm{mT}$ ) and (b) for best matching condition for each pressure/power.

known as the source relaxation oscillations. The second is a transport instability occurring downstream in the expanding chamber. While the source instability strongly modulates the plasma parameters in phase throughout the discharge, the downstream instability is related to a propagating phenomenon and has a maximum in amplitude at a given position. We showed, using time-resolved Langmuir probe measurements, that the downstream instability observed is the periodic formation and propagation of a double layer. The double-layer formation requires a certain fraction of negative ions in the discharge. Three regimes are observed: (i) when operating with pure argon or for low $\mathrm{SF}_{6}$ concentrations, the plasma potential decreases smoothly from the source to the diffusion chamber and no double layer DL is observed, (ii) for intermediate $\mathrm{SF}_{6}$ concentrations (namely, between $8 \%$ and $13 \%$ ), a stationary DL is formed and separates a highelectronegativity plasma downstream and a lowelectronegativity plasma upstream, and (iii) for high $\mathrm{SF}_{6}$ concentrations, the DL shows the propagating features described above, resulting in the "downstream instability." There are similarities between the downstream instability presented here and that presented by Tuszewski et al.; ${ }^{9}$ (i) the frequencies are similar, (ii) it is a propagating phenomenon born at a particular location, (iii) the propagating speed is in the same range ( $\approx 150 \mathrm{~m} \mathrm{~s}^{-1}$ in both experiments), (iv) the upstream plasma is somewhat compressed but shows small perturbations compared with the downstream, (v) the oscillations are fairly insensitive to the rf circuitry (matchbox, frequency, and antenna coupling), (vi) they can be suppressed when the length of the expanding region is reduced, and (vii) they were observed for comparable gas mixtures and pressures. However, there are also important differences between the two experiments. Most significantly is the difference in charged-particle dynamics: the electron flux appears to be highly modulated in the downstream region in our system, while Fig. 6 in Ref. 9 shows very little variation in the electron density compared with the positive-ion density. Tuszewski et al. also observed that the position of the critical layer at which the modulation appears remains in the diffusion chamber and moves downward as the rf power is increased, leading to a stable discharge when the power was sufficiently raised; we observed that the double layer is always born at the interface of the two chambers, or slightly in the source when the inner tube is present. Finally, the instability windows are quite different when the geometry is more comparable to that of Ref. 9 (i.e., with the inner tube inserted in the present case).

The physical processes leading to this periodic formation remain unclear. It is well established that double layers, or negative-ion fronts, can form in low-pressure electronegative discharges. $^{23-25}$ A necessary condition for double-layer formation is that the ion sound speed exceeds the local ion acoustic speed. In electronegative plasmas, the ion acoustic speed is significantly lower than that in electropositive plasmas, ${ }^{26}$ which may explain why the DL forms above a minimum $\mathrm{SF}_{6}$ fraction. The unsteady behavior of the DL is more difficult to explain. Tuszewski and Gary ${ }^{10}$ have derived a threshold in ion velocities above which an electronegative plasma becomes linearly unstable. We can postulate that unsteady DLs are formed when both thresholds (ion acoustic limit and linear stability) are overcome.

\section{ACKNOWLEDGMENTS}

The authors would like to thank Professor A. J. Lichtenberg and Professor R. W. Boswell for many useful discussions on various aspects of this work.

${ }^{1}$ M. Lieberman, A. Lichtenberg, and A. Marakhtanov, Appl. Phys. Lett. 75, 3617 (1999).

${ }^{2}$ P. Chabert, A. Lichtenberg, M. Lieberman, and A. Marakhtanov, Plasma Sources Sci. Technol. 10, 478 (2001).

${ }^{3}$ A. Lichtenberg, P. Chabert, M. Lieberman, and A. Marakhtanov, Bifurcation Phenomena in Plasmas (2001), p. 3.

${ }^{4}$ P. Chabert, H. Abada, J.-P. Booth, and M. Lieberman, J. Appl. Phys. 94, 76 (2003).

${ }^{5}$ P. Chabert, A. Lichtenberg, M. Lieberman, and A. Marakhtanov, J. Appl. Phys. 94, 831 (2003).

${ }^{6}$ C. Corr, P. Steen, and W. Graham, Plasma Sources Sci. Technol. 12, 265 (2003).

${ }^{7}$ M. Tuszewski and R. White, J. Appl. Phys. 94, 2858 (2003).

${ }^{8}$ A. Marakhtanov, M. Tuszewski, M. L. A. Lichtenberg, and P. Chabert, J. Vac. Sci. Technol. A 21, 1849 (2003).

${ }^{9}$ M. Tuszewski, R. White, and G. Wurden, Plasma Sources Sci. Technol. 12, 396 (2003).

${ }^{10}$ M. Tuszewski and S. P. Gary, Phys. Plasmas 10, 539 (2003).

${ }^{11}$ R. Boswell, Phys. Lett. 33A, 470 (1970).

${ }^{12}$ A. Cantin and R. Gagne, Appl. Phys. Lett. 30, 31 (1977).

${ }^{13}$ M. Hopkins and W. Graham, Rev. Sci. Instrum. 57, 2210 (1986).

${ }^{14}$ P. Chabert, T. Sheridan, R. Boswell, and J. Perrin, Plasma Sources Sci. Technol. 8, 561 (1999).

${ }^{15}$ T. Sheridan, P. Chabert, and R. Boswell, Plasma Sources Sci. Technol. 8, 457 (1999).

${ }^{16}$ F. Magnus and J. Gudmundsson, Internal Report, Science Institute, University of Iceland RH-20-2002 (2002).

${ }^{17}$ A. Savitzky and M. Golay, Anal. Chem. 44, 1627 (1964). 
${ }^{18}$ I. Sudit and R. Woods, Rev. Sci. Instrum. 64, 2440 (1993).

${ }^{19}$ H. M. Smith and I. Langmuir, Phys. Rev. 28, 727 (1926).

${ }^{20}$ C. Corr, P. Steen, and W. Graham, Appl. Phys. Lett. 86, 141503 (2005).

${ }^{21}$ N. Plihon, C. Corr, and P. Chabert, Appl. Phys. Lett. 86, 091501 (2005)

${ }^{22}$ J. Andrews and J. Allen, Proc. R. Soc. London, Ser. A 320, 459 (1971).
${ }^{23}$ I. Kouznetsov, A. Lichtenberg, and M. Lieberman, J. Appl. Phys. 86, 4142 (1999).

${ }^{24}$ I. Kaganovich, Phys. Plasmas 8, 2540 (2001).

${ }^{25}$ T. Sheridan, J. Phys. D 32, 1761 (1999).

${ }^{26}$ N. Braithwaite and J. Allen, J. Phys. D 21, 1733 (1988). 